\title{
PRIMES IN SEQUENCES ASSOCIATED TO POLYNOMIALS (AFTER LEHMER)
}

\section{MANFRED EINSIEDLER, GRAHAM EVEREST AND THOMAS WARD}

\begin{abstract}
In a paper of 1933, D. H. Lehmer continued Pierce's study of integral sequences associated to polynomials generalizing the Mersenne sequence. He developed divisibility criteria, and suggested that prime apparition in these sequences - or in closely related sequences would be denser if the polynomials were close to cyclotomic, using a natural measure of closeness.

We review briefly some of the main developments since Lehmer's paper, and report on further computational work on these sequences. In particular, we use Mossinghoff's collection of polynomials with smallest known measure to assemble evidence for the distribution of primes in these sequences predicted by standard heuristic arguments.

The calculations lend weight to standard conjectures about Mersenne primes, and the use of polynomials with small measure permits much larger numbers of primes to be generated than in the Mersenne case.
\end{abstract}

\section{Introduction}

Let $f \in \mathbb{Z}[x]$ be a monic polynomial with factorization

$$
f(x)=\left(x-\alpha_{1}\right) \ldots\left(x-\alpha_{d}\right)
$$

over the complex numbers. Following Pierce [19] and Lehmer [12], define a sequence of integers by

$$
\Delta_{n}(f)=\prod_{i=1}^{d}\left|\alpha_{i}^{n}-1\right| .
$$

For example, if $f(x)=x-2$, then $\Delta_{n}(f)=2^{n}-1$ is the classical Mersenne sequence. Pierce and Lehmer studied the possible factors of $\Delta_{n}(f)$, and Lehmer in particular used these results to compute large primes. For our purposes, the detailed arguments concerning possible factors are not relevant, but three key observations by Lehmer are:

1. if $\left|\alpha_{i}\right| \neq 1$ for $i=1, \ldots, d$ then $\Delta_{n}(f) / \Delta_{n-1}(f) \rightarrow M(f)=\prod_{i:\left|\alpha_{i}\right|>1}\left|\alpha_{i}\right|$;

2. if $M(f)$ is close to 1 , then $\Delta_{n}(f)$ may be expected to be prime often;

3. prime factors of $\Delta_{n}$ satisfy (essentially) linear congruences.

It is clear from Kronecker's lemma that $M(f)=1$ if and only if $f$ is cyclotomic. Lehmer made an extensive search for non-cyclotomic polynomials with measure close to 1 , and 
his example of degree 10 (referred to below as $f_{1}$ ) with $M(f)=1.176 \ldots$ is still the closest known. He also made the prescient remark that for non-cyclotomic polynomials, a zero on the unit circle 'contributes an oscillating factor which, although it never vanishes or becomes infinite, cannot be estimated readily' and went on to use $M(f)$ as a natural measure of growth in this case also (cf. the convergence (3), discovered later).

Many subsequent authors have shed new light on various aspects of the sequence $\left(\Delta_{n}(f)\right)$ and the associated growth rate $M(f)$. Mahler [15] pointed out that Jensen's formula gives the integral form

$$
m(f)=\log M(f)=\int_{0}^{1} \log \left|f\left(e^{2 \pi i t}\right)\right| \mathrm{d} t
$$

for the measure, which is now called the (logarithmic) Mahler measure of $f$. A huge amount of work has gone into attempts to resolve Lehmer's problem: are there polynomials with arbitrarily small positive logarithmic measure? For an overview of this circle of results from a theoretical perspective, see [2] and [8]. The view of polynomials with small measure as being small perturbations of cyclotomic ones is explored in [17]. For recent results on computations of Mahler measures and their connections with other parts of mathematics, see [4], [7], [14] and [16].

To each polynomial of the form (1) there is an associated endomorphism $T_{f}$ of the $d$ torus, given by the natural action of the companion matrix of $f$. If no zero of $f$ is a root of unity, then $T_{f}$ is an ergodic transformation with respect to Lebesgue measure, and $\Delta_{n}(f)$ is the number of points of period $n$ under $T_{f}$. Expansiveness of $T_{f}$ as a topological dynamical system corresponds to Lehmer's condition that $\left|\alpha_{i}\right| \neq 1$ for $i=1, \ldots, d$. Finally, the topological entropy of $T_{f}$ is equal to $m(f)$. This links arithmetic properties of the sequence to dynamical properties of the corresponding toral endomorphism - see [13]. Accordingly, we call the polynomial $f$ expansive if $\left|\alpha_{i}\right| \neq 1$ for $i=1, \ldots, d$, ergodic if no $\alpha_{i}$ is a root of unity, and quasihyperbolic if it is ergodic but not expansive.

Finally, the convergence observed by Lehmer in the expansive case does not extend to the quasihyperbolic case (see [6,8, Theorem 2.16], but the more robust convergence

$$
\frac{1}{n} \log \Delta_{n}(f) \longrightarrow m(f)
$$

extends to the quasihyperbolic case by Gelfond's Diophantine results (see [9] and [13]). Some measure of the Diophantine subtlety involved in convergence (3) may be seen in the sequence corresponding to $f_{1}$ (defined below): $\Delta_{n}\left(f_{1}\right)$ behaves asymptotically like $(1.176 \ldots)^{n}$ but $\Delta_{n}\left(f_{1}\right)=1$ for values of $n$ as large as 74 . These dramatically small values for relatively large values of $n$ are reflected in the graphs below by the irregular early behaviour.

\section{Arithmetic of $\Delta_{n}$}

The polynomial (1) is said to be reciprocal if $x^{d} f\left(x^{-1}\right)=f(x)$. Boyd $[\mathbf{1}, \mathbf{3}]$ and Mossinghoff [16] have carried out extensive calculations of Mahler measures; from [16] we use the list of the 100 irreducible polynomials with smallest known positive Mahler measure. These are all reciprocal (a beautiful result of Smyth [21] shows that if $f$ is nonreciprocal and $f(0) f(1) \neq 0$, then $\left.m(f) \geqslant m\left(x^{3}-x-1\right)=0.281 \ldots\right)$, and are known to divide polynomials with coefficients in $\{0, \pm 1\}$. If $f$ is a reciprocal polynomial, then $\Delta_{n}(f) / \Delta_{1}(f)$ is a perfect square for $n$ odd, by the following argument. If $\alpha$ is a zero of $f$, 
let $K=\mathbb{Q}(\alpha)$ and $K^{\prime}=\mathbb{Q}\left(\alpha+\alpha^{-1}\right)$. Then

$$
\begin{aligned}
\Delta_{n}(f) & =\left|N_{K / \mathbb{Q}}\left(\alpha^{n}-1\right)\right| \\
& =\left|N_{K / \mathbb{Q}}(\alpha-1) N_{K / \mathbb{Q}}\left(1+\alpha+\ldots+\alpha^{n-1}\right)\right| \\
& =\Delta_{1}(f) \times\left|N_{K / \mathbb{Q}}\left(\alpha^{(n-1) / 2}\right) N_{K / \mathbb{Q}}\left(\alpha^{-(n-1) / 2}+\ldots+\alpha^{(n-1) / 2}\right)\right| .
\end{aligned}
$$

Now $\xi=\alpha^{-(n-1) / 2}+\ldots+\alpha^{(n-1) / 2}$ is an integral element of $K^{\prime}$, so

$$
N_{K / \mathbb{Q}}(\xi)=\left(N_{K^{\prime} / \mathbb{Q}}(\xi)\right)^{2}
$$

is a square. Accordingly, define $\Gamma_{n}(f)$ by $\Gamma_{n}(f)^{2}=\Delta_{n}(f) / \Delta_{1}(f)$ for odd $n \geqslant 1$.

Prime values of $\Gamma_{n}(f)$ may arise for composite values of $n$, and such values are called anomalous. In the expansive case it is clear that the anomalous primes are finite in number, and this remains so in the quasihyperbolic case, for a deeper reason.

Proposition 1. If $f$ is an ergodic polynomial, then there are only finitely many anomalous primes in the sequence $\left(\Gamma_{n}(f)\right)$, or in $\left(\Delta_{n}(f) / \Delta_{1}(f)\right)$ in the non-reciprocal case.

Proof. First notice that the sequence is multiplicative. Write $M=M(f)^{1 / 2}$ for the square root of the Mahler measure of $f$, and $\Gamma_{n}$ for $\Gamma_{n}(f)$. (Note that a similar argument holds for $\left(\Delta_{n}(f) / \Delta_{1}(f)\right)$ in the non-reciprocal case.) By Baker's theorem (see [8] for references), there are constants $A, B, C>0$ with

$$
A M^{n}>\Gamma_{n}>B M^{n} / n^{C} .
$$

It follows that only finitely many $n$ can have $\Gamma_{n}=1$.

Now an anomalous prime occurs when $\Gamma_{m n}$ is prime with $m, n>1$. If $\Gamma_{m}$ and $\Gamma_{n}$ are both 1 , then $m$ and $n$ are bounded by the previous paragraph. On the other hand,

$$
\Gamma_{m n} / \Gamma_{m}>B M^{m n} / A(m n)^{C} M^{m}=D M^{n(m-1)} /(m n)^{C} .
$$

If the left-hand-side is 1 , then there is an upper bound of the form

$$
E+F(\log n+\log m)
$$

for $n(m-1)$, which bounds both $m$ and $n$.

This precludes $\Gamma_{m n}=\Gamma_{m}$ for all but finitely many $m$ and $n$.

Recall that $K$ is the field defined by the chosen irreducible polynomial $f$, and let

$$
\begin{aligned}
h_{K} & =\text { class number of } K \\
r_{1} & =\text { the number of real embeddings of } K ; \\
r_{2} & =\text { half the number of complex embeddings of } K ; \\
w_{K} & =\text { the number of unit roots in } K ; \\
R_{K} & =\text { the regulator of } K ; \\
d_{K} & =\text { the discriminant of } f ; \\
\rho_{K} & =\frac{2^{r_{1}}(2 \pi)^{r_{2}} h_{K} R_{K}}{w_{K} \sqrt{\left|d_{K}\right|}} .
\end{aligned}
$$

Define as usual the Dedekind zeta-function for $K$ by

$$
\zeta_{K}(s)=\sum_{\mathfrak{q}} \frac{1}{N_{K / \mathbb{Q}}(\mathfrak{q})^{s}},
$$


where $\mathfrak{q}$ runs through the ideals of $O_{K}$, with Laurent expansion at $s=1$ given by

$$
\zeta_{K}(s)=\frac{\rho_{K}}{s-1}+\gamma_{K}+\ldots
$$

and Euler product form

$$
\zeta_{K}(s)=\prod_{\mathfrak{p}}\left(1-\frac{1}{N_{K / \mathbb{Q}}(\mathfrak{p})^{s}}\right)^{-1}
$$

where $\mathfrak{p}$ runs through the prime ideals of $O_{K}$. Finally, there is the number-field analogue of Merten's theorem (see [10], [11] or [20]).

\section{Proposition 2.}

$$
\sum_{N_{K / \mathbb{Q}}(\mathfrak{p}) \leqslant x}-\log \left(1-\frac{1}{N_{K / \mathbb{Q}}(\mathfrak{p})}\right)=\log \log x+\gamma+\log \rho_{K}+O(1 / \log x)
$$

where $\mathfrak{p}$ runs through the prime ideals of $O_{K}$, and $\gamma=0.577 \ldots$ is the classical Euler constant.

\section{Heuristic arguments}

The Mersenne numbers $M_{n}=2^{n}-1$ are well-known, and 38 values of $n$ are known for which $M_{n}$ is prime. An elegant probabilistic argument due to Wagstaff [22] gives the following expected distribution of prime values of $M_{n}$. If $n_{1}, n_{2}, \ldots$ are the primes for which $M_{n_{j}}$ is prime, then $j / \log _{2} \log _{2} M_{n_{j}}$ is conjectured to converge to a constant. This is a consequence of the simple linear congruences satisfied by factors of $M_{n}$ (from the Euler-Fermat theorem), and Merten's theorem.

In the Lehmer case, essentially the same argument may be applied, but the arithmetic of the sequence and the analytic properties of the corresponding zeta function are more involved. The calculations described below give the following results.

1. There is compelling numerical evidence to suggest that

$$
\frac{j}{\log \log \Gamma_{n_{j}}} \longrightarrow E_{f}
$$

for some positive limit $E_{f}$ as $j \rightarrow \infty$, where $n_{1}, n_{2}, \ldots$ is the sequence of prime indices for which $\Gamma_{n_{j}}$ is prime.

2. A naive number-field analogue of Wagstaff's heuristics suggests that $E_{f}$ is given by $W_{f}=2 e^{\gamma} / m(f)$, which is compatible with the numerical evidence.

3. The more subtle quantity $C_{f}=2 e^{\gamma_{K^{\prime}}} / m(f)$ (or $2 e^{\gamma_{K}} / m(f)$ in the non-reciprocal case) is sometimes closer to the observed $E_{f}$, though we do not have a heuristic rationale for this, and the calculation of $\gamma_{K^{\prime}}$ (or $\gamma_{K}$ ) itself presents considerable difficulties for extensions of large degree.

4. The discrepancy between the observed value of $E_{f}$ and either of the heuristic constants is substantial enough to suggest that more subtle arithmetic phenomena are at work.

To explain the heuristic argument, we follow essentially Caldwell's exposition of the Wagstaff heuristics (available on the WWW 'Prime Pages' site — see [5]). Assume that $p$ is prime. If $\mathfrak{p}$ is a prime ideal in $O_{K}$ with

$$
N_{K / \mathbb{Q}}(\mathfrak{p}) \mid N_{K / \mathbb{Q}}\left(\alpha^{p}-1\right)
$$


then $N_{K / \mathbb{Q}}(\mathfrak{p}) \equiv 1 \bmod p$. It follows that the probability of $\Gamma_{p}(f)$ being prime is increased by the ratio $N_{K / \mathbb{Q}}(\mathfrak{p}) /\left(N_{K / \mathbb{Q}}(\mathfrak{p})-1\right)$ for each prime ideal $\mathfrak{p}$ of $O_{K}$ with prime norm $N_{K / \mathbb{Q}}(\mathfrak{p}) \leqslant p$. The set

$$
\left\{\mathfrak{r} \mid \mathfrak{r} \text { is an ideal of } O_{K} \text { with } N_{K / \mathbb{Q}}(\mathfrak{r}) \leqslant x\right\}
$$

has asymptotically $\rho_{K} x$ members, of which $x / \log x$ are prime ideals with prime norm. It follows that the probability that an integral ideal $\mathfrak{r}$ is a prime ideal with prime norm in $O_{K}$ is $1 /\left(\rho_{K} \log N_{K / \mathbb{Q}}(\mathfrak{r})\right)$.

In the Mersenne case, the resulting product is estimated using Merten's theorem; here we use Proposition 2 instead. The discussion above suggests that the probability that $\Gamma_{p}(f)$ is prime is approximately

$$
\begin{aligned}
P_{f}(p) & =\left(\frac{2 \rho_{K}^{-1}}{p m(f)}\right) \prod_{N_{K / \mathbb{Q}}(\mathfrak{p}) \leqslant p}\left(\frac{N_{K / \mathbb{Q}}(\mathfrak{p})}{\left(N_{K / \mathbb{Q}}(\mathfrak{p})-1\right)}\right) \\
& =\left(\frac{2 \rho_{K}^{-1}}{p m(f)}\right)\left(e^{\gamma} \rho_{K} \log p+O(1 / p)\right) .
\end{aligned}
$$

So the expected number of (non-anomalous) prime values of $\Gamma_{p}(f)$ with $p \leqslant x$ is given by ( $p$ running through the rational primes)

$$
\begin{aligned}
\sum_{p \leqslant x} P_{f}(p) & =\frac{2 \rho_{K}^{-1}}{m(f)} \sum_{p \leqslant x} \frac{1}{p} \cdot \prod_{N_{K / \mathbb{Q}}(\mathfrak{p}) \leqslant p}\left(\frac{N_{K / \mathbb{Q}}(\mathfrak{p})}{N_{K / \mathbb{Q}}(\mathfrak{p})-1}\right) \\
& \sim \frac{2 e^{\gamma}}{m(f)}\left(\sum_{p \leqslant x} \frac{\log p}{p}\right) \\
& \sim\left(\frac{2 e^{\gamma}}{m(f)}\right) \log x .
\end{aligned}
$$

Notice that in the Mersenne case, the sum is taken over all $n$, weighted according to the probability that $n$ is prime; summing instead over primes $p$ without weighting, as we have done here, gives the same estimate.

If we write $n_{1}, n_{2}, \ldots$ for the sequence of indices for which $\Gamma_{n_{j}}$ is prime, this suggests that the number of prime values of $\Gamma_{n_{j}}$ with $n_{j} \leqslant x$ is approximately $\left(2 e^{\gamma} / m(f)\right) \log x$. It follows that

$$
\frac{\log \log \Gamma_{n_{j}}}{j} \rightarrow \frac{m(f)}{2 e^{\gamma}} .
$$

Notice that the effect of any further congruence conditions on possible factors of $\Gamma_{n}(f)$ will be to asymptotically increase the number of primes appearing in the sequence, so the relationship

$$
E_{f} \geqslant \frac{2 e^{\gamma}}{m(f)}
$$

between convergences (7) and (8) is expected. However, the results shown in Table 2 do not give a consistent inequality; if anything, they suggest the reverse (see Section 6).

In the case of non-reciprocal polynomials, the factor 2 (which came from the fact that $\Gamma_{n}$ is logarithmically half of $\Delta_{n}$ ) needs to be removed, so for non-reciprocal $f$ the letters $E_{f}, W_{f}, C_{f}$ will be used for the analogous quantities also. 
Three questions were therefore examined numerically. Firstly, is the sequence associated to a polynomial with small Mahler measure very rich in primes? Secondly, do calculations suggest the distribution (7) for prime apparition in these sequences with some limiting constant? Thirdly, does the 'limiting constant' observed lend support to the heuristic argument?

The results are - unsurprisingly — mixed. The first question can be answered with an emphatic 'yes': in a short search on modest equipment, sequences have been found containing over one hundred primes. The second is answered with an equivocal 'yes': the analogous plots for the polynomials of small measure do look linear (details of the statistical method used are given below). The third question probably requires a deeper understanding of the arithmetic of $\Gamma_{n}$, but the numbers agree fairly well. In particular, the number of primes found does decrease as the Mahler measure increases.

In light of this, it would be of interest to find a reformulation of the Mersenne heuristics in which $\gamma$ appears, not via Merten's Theorem, but as the second coefficient of the Laurent expansion of the Riemann zeta function at $s=1$.

A feature of this work is that the use of polynomials with very small measure gives significant data on Mersenne-like problems without the difficulty of testing excessively large numbers for primality. The idea of using polynomials with small measure in this way comes directly from Lehmer's paper.

\section{Description of the calculations}

Given a candidate polynomial $f$ with small Mahler measure, the prime values of $n$ for which $\Gamma_{n}(f)$ is prime up to some limit were computed. Composite values of $n$ for which $\Gamma_{n}(f)$ is prime give rise to the anomalous primes. Primality testing was for pseudo-primality to ten randomly chosen bases: in particular, the lack of an analogue of the Lucas-Lehmer test means that the primality test used is the Miller-Rabin test. Thus, in this paper, prime values of $\Gamma_{n}$ or $\Delta_{n} / \Delta_{1}$ are probable primes. All the calculations were done using PARI-GP; see [18] for more details.

For the first two polynomials in the Mossinghoff list,

$$
f_{1}(x)=x^{10}+x^{9}-x^{7}-x^{6}-x^{5}-x^{4}-x^{3}+x+1
$$

and

$f_{2}(x)=x^{18}+x^{17}+x^{16}+x^{15}-x^{12}-x^{11}-x^{10}-x^{9}-x^{8}-x^{7}-x^{6}+x^{3}+x^{2}+x+1$,

the calculations were performed for $n$ up to 200000 . For each of the remaining polynomials $f_{3}, \ldots, f_{40}$, ranging in degree from 10 to 52 , the calculations were performed for $n$ up to 50000 . The full list of polynomials is in the paper [16]. In order to gain more insight into how much of the prime behaviour is governed simply by the field arithmetic, the same calculation was also carried out for the 'negative' polynomials, $f_{j}^{-}(x)=f_{j}(-x)$.

The constant $\gamma_{K^{\prime}}$ has also been computed in some cases (as has $\gamma_{K}$ in some non-reciprocal cases), though this requires extensive calculation itself. The method adopted is to use the Laurent expansion (5) and estimate $\gamma_{K^{\prime}}=\lim _{s \rightarrow 1^{+}}\left((s-1) \zeta_{K^{\prime}}(s)-\rho_{K^{\prime}}\right)$, with $K$ replacing $K^{\prime}$ in the non-reciprocal case, using GP's ability to compute values of the Dedekind zeta functions for number fields of small degree.

The empirical constant $E_{f}$ is found using a least-squares linear regression. 


\section{Results}

We present several graphs of $\log \log \Gamma_{n_{j}}(f)$ against $j$, which indicate the asymptotic linearity (Figures 1-9). On each graph, the number on the abscissa is the total number of non-anomalous primes found for that polynomial. In each case the values of $C_{f}, W_{f}$ and $E_{f}$ are given. The graphs have been chosen from the (small) sample of polynomials for which $\gamma_{K}$ (or $\gamma_{K^{\prime}}$ ) can be computed. As mentioned above, the non-reciprocal polynomials do not have the factor 2 in the expressions for $W$ and $C$. The numerical constants have been rounded to three decimal places.

Table 1 gives some data for the Mersenne case, some simple non-reciprocal polynomials, and for those $f_{ \pm j}$ for which $C$ could be computed (the polynomial $f_{2}$, of degree 18 , is included here despite the fact that we have been unable to compute $C_{f_{2}}$ ). For the nonreciprocals, the growth rate is much higher by Smyth's result, and so the calculations are limited. In addition to the Mersenne case and some polynomials from [16] for which $C$ could be found, some non-reciprocal polynomials of small height have been chosen. These non-reciprocal polynomials are those with smallest Mahler measure in the list of irreducible non-cyclotomic factors of trinomials with smallest known Mahler measures - we thank David Boyd for providing this list of trinomials. Table 1 is thus a mixed bag of polynomials selected on the basis of having small measure for polynomials of a certain shape, or for being of relatively small degree. Table 1 records

1. the polynomial $f$;

2. the Mahler measure $M(f)$;

3. the range searched, $1 \leqslant n \leqslant R$;

4. the number $N$ of non-anomalous primes found;

5. the empirical constant $E_{f}$ found using least-squares;

6. the heuristic constant $W_{f}$;

7. the heuristic constant $C_{f}$.

The polynomials in Table 1 are arranged in order of increasing Mahler measure.

Table 2 summarises the bulk of our results. It lists the following quantities:

1. the number $j$ of the polynomial in the list from [16];

2. the Mahler measure $M\left(f_{j}^{ \pm}\right)$;

3. $N\left(f_{j}^{ \pm}\right)$, the number of non-anomalous prime values of $\Gamma_{n}\left(f_{j}^{ \pm}\right)$;

4. $E_{f_{j}^{ \pm}}$, the least-squares estimate;

5. $W_{f_{j}^{ \pm}}$, the value computed using the heuristic argument above.

The polynomials are again arranged in order of increasing Mahler measure.

\section{Open problems}

Several problems are suggested by this work, of which the most pressing seem to be the following. What is behind the examples in which $E_{f}$ is smaller than $W_{f}$ ? Can a heuristic argument be found that predicts $E_{f}$ with the same level of accuracy as that seen in the Mersenne case? In particular, significant differences between $E_{f_{j}}$ and $E_{f_{j}^{-}}$in Table 2 suggest that more accurate heuristics must involve the polynomial itself, and cannot depend only on the arithmetic of the field defined by the polynomial. 


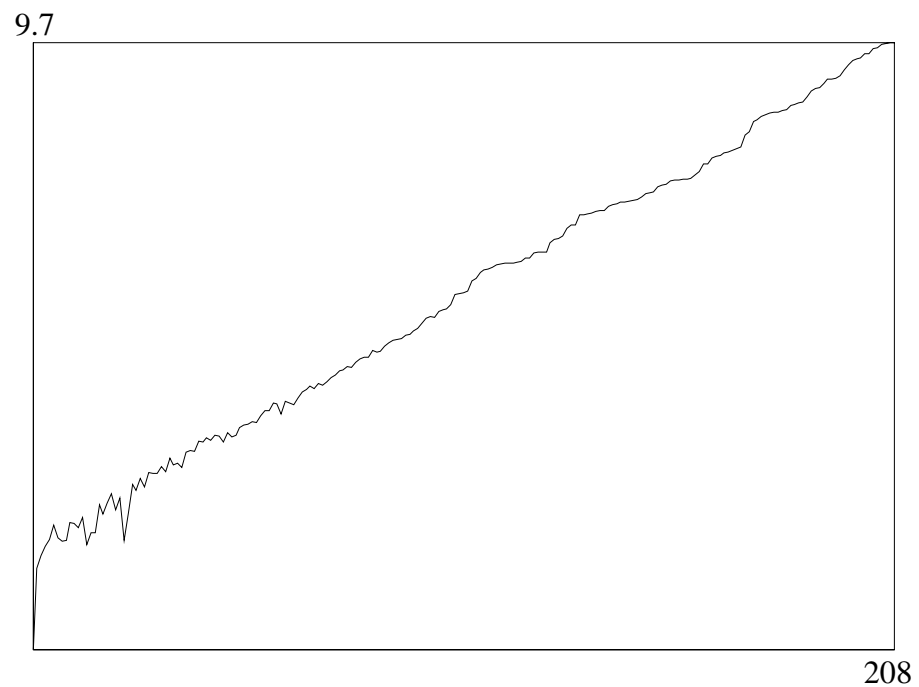

Figure 1: Graph of $\log \log \Gamma_{n_{j}}\left(f_{1}\right)$ against $j$ for $n \leqslant 200,000$; $E_{f_{1}}=25.719, W_{f_{1}}=21.949, C_{f_{1}}=24.767$.

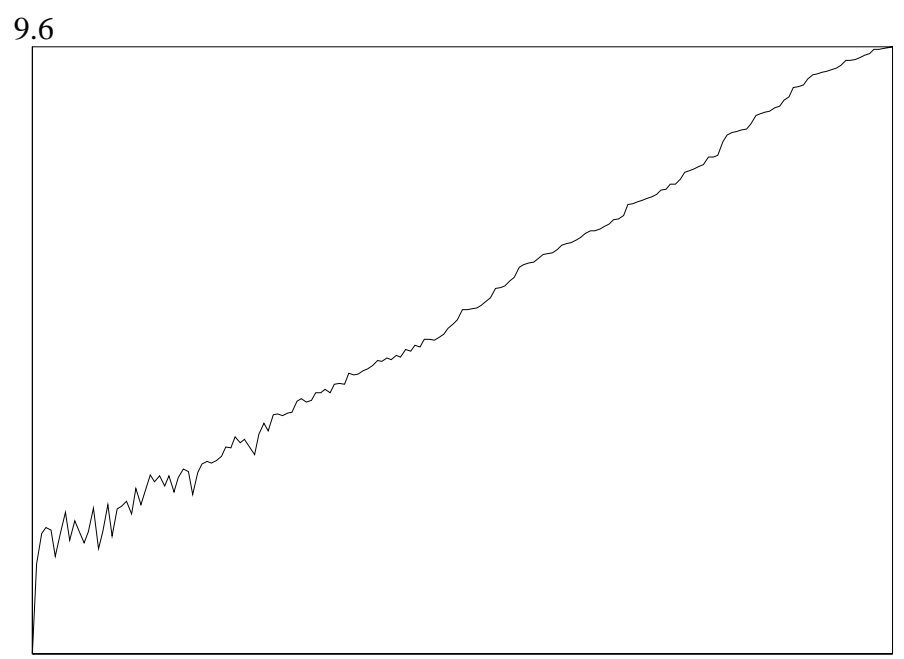

182

Figure 2: Graph of $\log \log \Gamma_{n_{j}}\left(f_{2}\right)$ against $j$ for $n \leqslant 200,000$; $E_{f_{1}}=21.852, W_{f_{1}}=20.640$. 


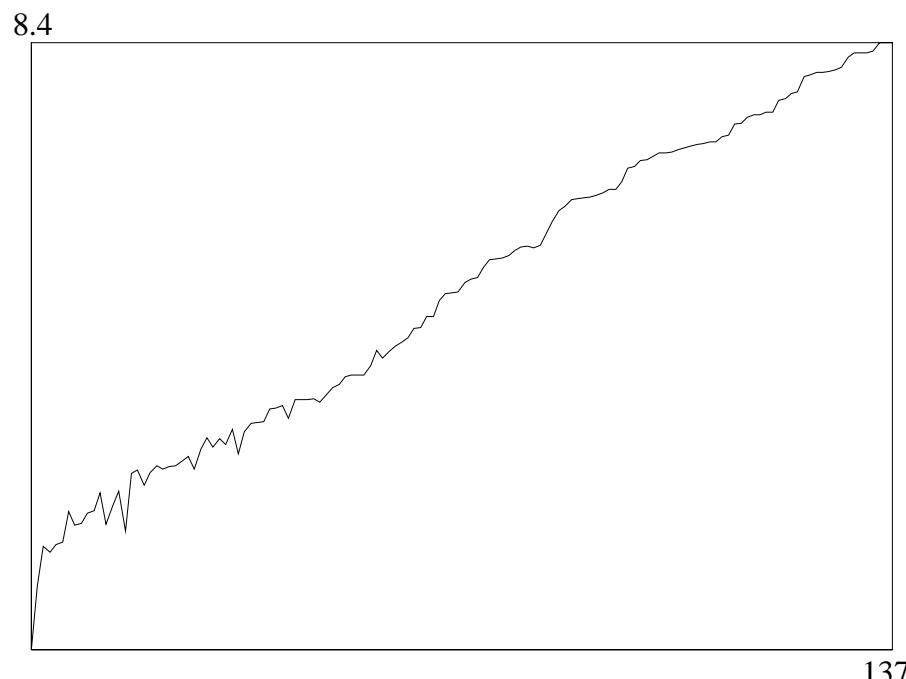

Figure 3: Graph of $\log \log \Gamma_{n_{j}}\left(f_{10}\right)$ against $j$ for $n \leqslant 50,000$; $E_{f_{10}}=18.507, W_{f_{10}}=18.191, C_{f_{10}}=18.844$.

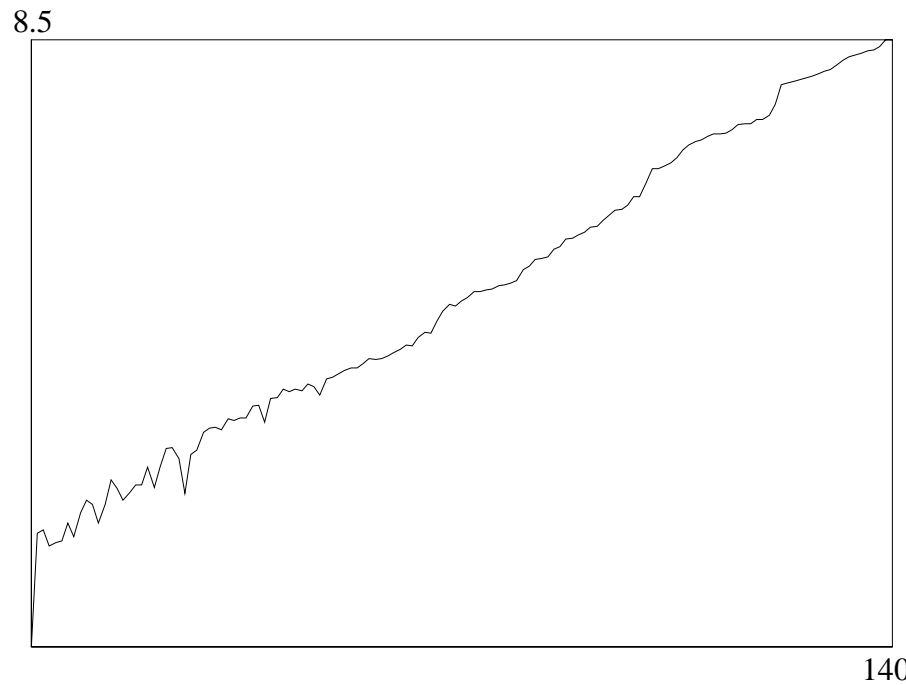

Figure 4: Graph of $\log \log \Gamma_{n_{j}}\left(f_{26}\right)$ against $j$ for $n \leqslant 50,000$; $E_{f_{26}}=19.384, W_{f_{26}}=17.364, C_{f_{26}}=18.782$. 
Primes in sequences associated to polynomials (after Lehmer)

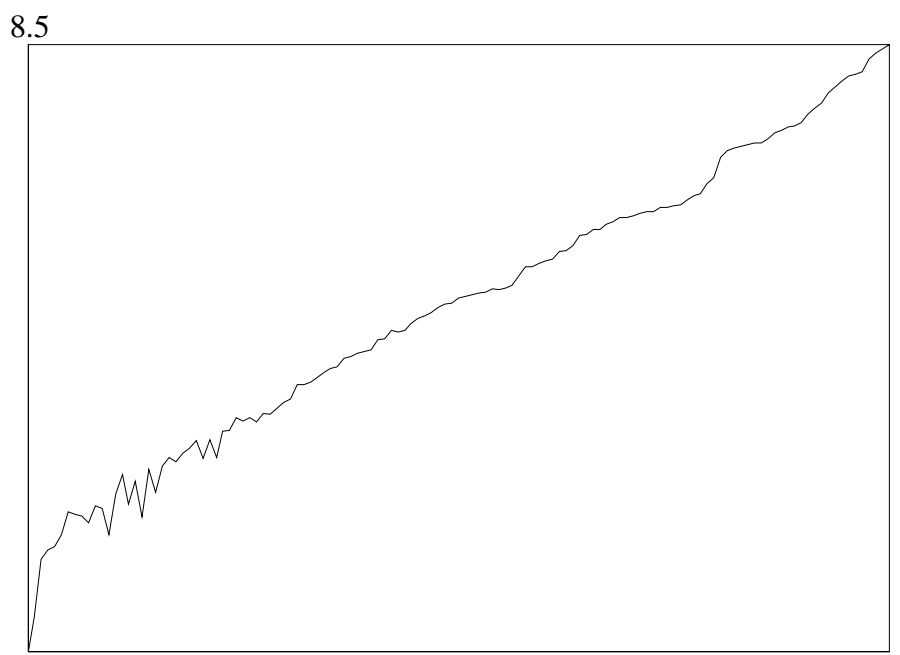

128

Figure 5: Graph of $\log \log \Gamma_{n_{j}}\left(f_{33}\right)$ against $j$ for $n \leqslant 50,000$; $E_{f_{33}}=18.984, W_{f_{33}}=17.187, C_{f_{33}}=17.869$.

8.5

Figure 6: Graph of $\log \log \Gamma_{n_{j}}\left(x^{3}-x-1\right)$ against j for $n \leqslant 20,000$; $E_{f}=6.807, W_{f}=6.334, C_{f}=6.398$. 


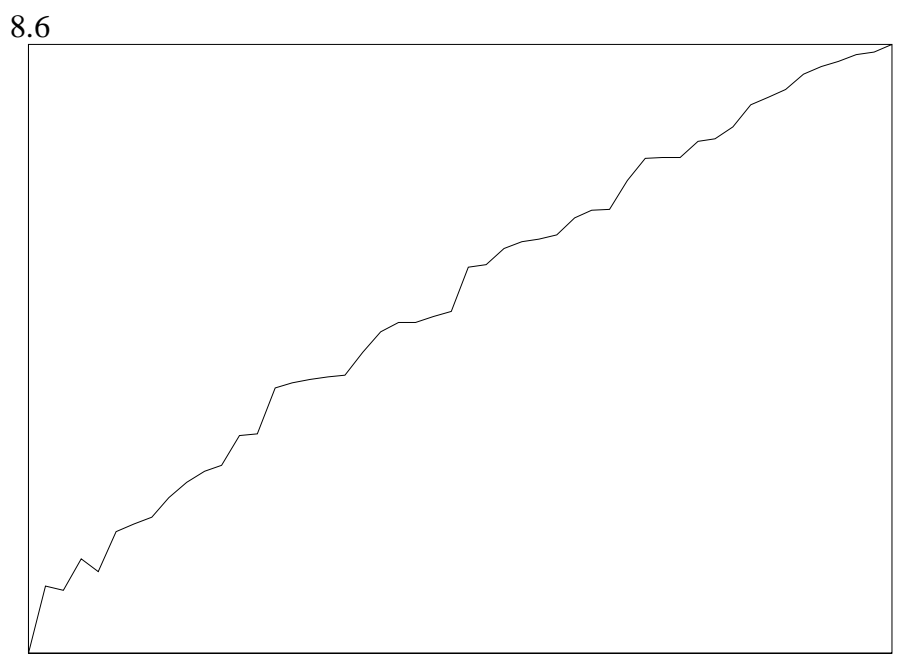

Figure 7: Graph of $\log \log \Gamma_{n_{j}}\left(x^{5}-x^{4}+x^{2}-x+1\right)$ against $j$ for $n \leqslant 20,000 ; E_{f}=6.128, W_{f}=5.939, C_{f}=5.930$.

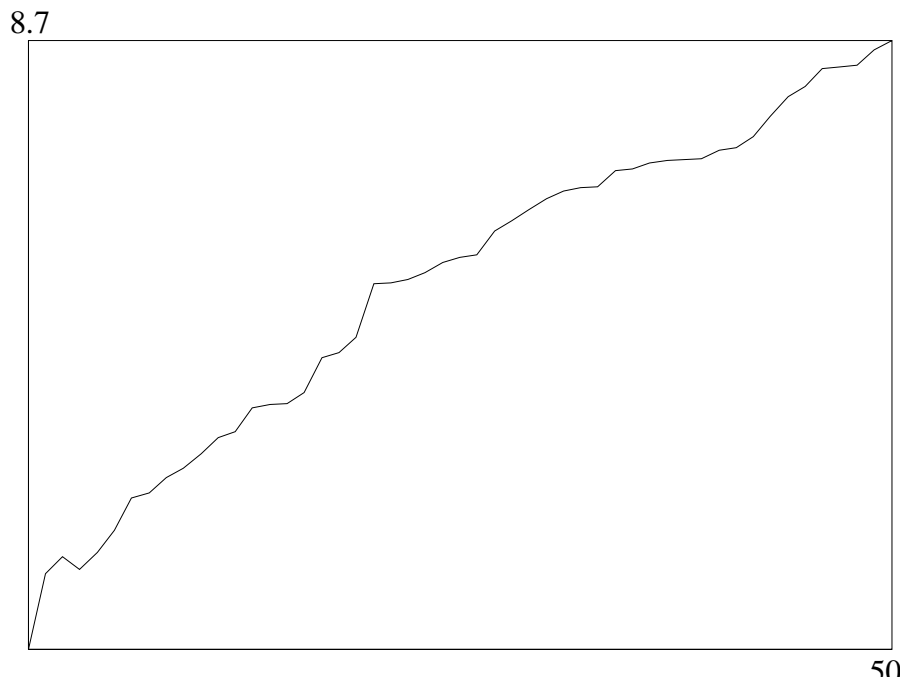

Figure 8: Graph of $\log \log \Gamma_{n_{j}}\left(x^{6}-x^{5}+x^{3}-x^{2}+1\right)$ against $j$ for $n \leqslant 20,000 ; E_{f}=6.519, W_{f}=5.793, C_{f}=5.942$. 


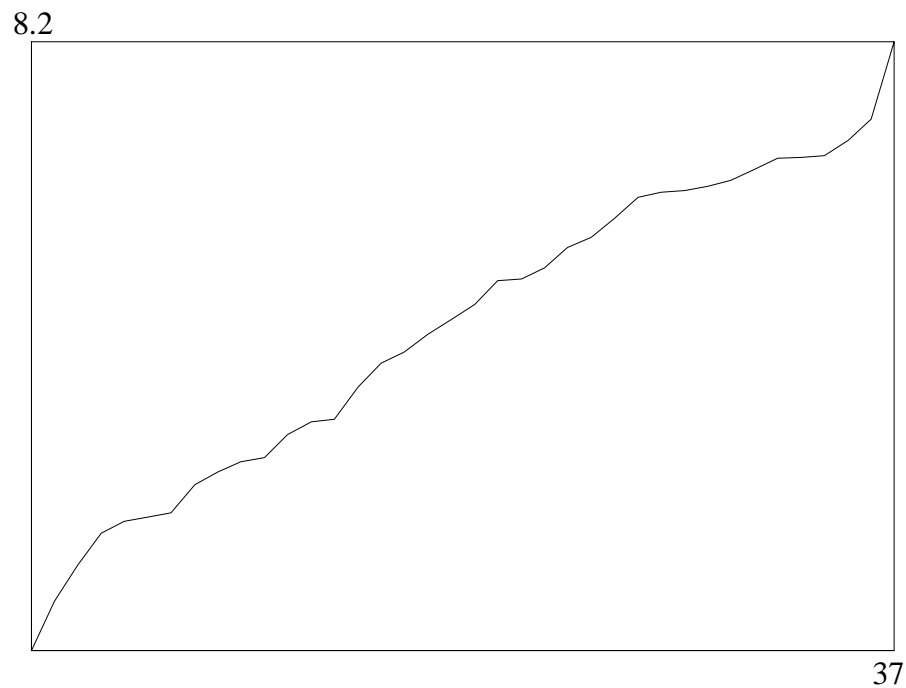

Figure 9: Graph of $\log \log \Gamma_{n_{j}}\left(x^{5}+x^{2}-1\right)$ against $j$ for $n \leqslant$ 20,$000 ; E_{f}=5.411, W_{f}=5.735, C_{f}=5.968$.

Table 1: Mahler measure $M$, numbers $N$ of prime values of $\Gamma_{n}$ or $\Delta_{n}$ found for $n \leqslant R$, empirical constant $E$, and two heuristic constants $W$ and $C$ for selected polynomials.

\begin{tabular}{crrrrrr}
\hline$f$ & $M(f)$ & $R$ & $N$ & $E_{f}$ & $W_{f}$ & $C_{f}$ \\
\hline$f_{1}$ & 1.176 & 200,000 & 208 & 25.719 & 21.940 & 24.767 \\
$f_{2}$ & 1.188 & 200,000 & 182 & 21.852 & 20.640 & \\
$f_{10}$ & 1.216 & 50,000 & 137 & 18.507 & 18.184 & 18.884 \\
$f_{-10}$ & 1.216 & 50,000 & 133 & 18.219 & 18.184 & 18.884 \\
$f_{26}$ & 1.227 & 50,000 & 140 & 19.384 & 17.358 & 18.782 \\
$f_{-26}$ & 1.227 & 50,000 & 145 & 21.297 & 17.358 & 18.782 \\
$f_{33}$ & 1.230 & 50,000 & 128 & 18.984 & 17.180 & 17.869 \\
$f_{-33}$ & 1.230 & 50,000 & 132 & 18.083 & 17.180 & 17.869 \\
$x^{3}-x-1$ & 1.325 & 20,000 & 47 & 6.807 & 6.334 & 6.398 \\
$x^{3}-x^{2}+1$ & 1.325 & 20,000 & 46 & 5.963 & 6.334 & 6.398 \\
$x^{5}-x^{4}+x^{2}-x+1$ & 1.350 & 20,000 & 49 & 6.128 & 5.939 & 5.930 \\
$x^{5}+x^{4}-x^{2}-x-1$ & 1.350 & 20,000 & 51 & 6.479 & 5.939 & 5.930 \\
$x^{6}-x^{5}+x^{3}-x^{2}+1$ & 1.360 & 20,000 & 50 & 6.519 & 5.793 & 5.942 \\
$x^{6}+x^{5}-x^{3}-x^{2}+1$ & 1.360 & 20,000 & 51 & 7.474 & 5.793 & 5.942 \\
$x^{5}+x^{2}-1$ & 1.364 & 20,000 & 37 & 5.411 & 5.735 & 5.968 \\
$x-2$ & 2 & $3,021,377$ & 37 & 2.549 & 2.569 & 2.569 \\
\hline
\end{tabular}


Table 2: Number $N$ of prime values of $\left(\Gamma_{n}\right)$, Mahler measure $M$, empirical constant $E$, and heuristic constant $W$ for polynomials $f_{1}, \ldots, f_{40}$ and $f_{1}^{-}, \ldots, f_{40}^{-}, n \leqslant 50,000$.

\begin{tabular}{|c|c|c|c|c|c|c|}
\hline$j$ & $M\left(f_{j}\right)=M\left(f_{j}^{-}\right)$ & $N\left(f_{j}\right)$ & $E_{f_{j}}$ & $W_{f_{j}}=W_{f_{j}^{-}}$ & $E_{f_{j}^{-}}$ & $N\left(f_{j}^{-}\right)$ \\
\hline 1 & 1.1762 & 173 & 25.899 & 21.940 & 23.493 & 166 \\
\hline 2 & 1.1883 & 151 & 22.482 & 20.640 & 23.420 & 156 \\
\hline 3 & 1.2000 & 137 & 18.912 & 19.535 & 19.724 & 133 \\
\hline 4 & 1.2013 & 171 & 24.618 & 19.413 & 20.803 & 146 \\
\hline 5 & 1.2026 & 126 & 19.644 & 19.307 & 22.004 & 155 \\
\hline 6 & 1.2050 & 148 & 21.374 & 19.100 & 18.356 & 132 \\
\hline 7 & 1.2079 & 128 & 18.211 & 18.854 & 21.109 & 144 \\
\hline 8 & 1.2128 & 136 & 19.127 & 18.461 & 18.905 & 136 \\
\hline 9 & 1.2149 & 145 & 22.572 & 18.291 & 18.542 & 128 \\
\hline 10 & 1.2163 & 137 & 18.507 & 18.184 & 18.219 & 133 \\
\hline 11 & 1.2183 & 134 & 19.974 & 18.032 & 19.211 & 135 \\
\hline 12 & 1.2188 & 135 & 18.619 & 17.998 & 19.594 & 140 \\
\hline 13 & 1.2190 & 122 & 16.996 & 17.983 & 19.885 & 135 \\
\hline 14 & 1.2194 & 114 & 16.258 & 17.954 & 21.704 & 151 \\
\hline 15 & 1.2197 & 137 & 18.941 & 17.934 & 17.399 & 130 \\
\hline 16 & 1.2202 & 115 & 16.667 & 17.892 & 16.919 & 124 \\
\hline 17 & 1.2234 & 145 & 20.884 & 17.663 & 19.529 & 136 \\
\hline 18 & 1.2237 & 136 & 18.806 & 17.639 & 15.666 & 113 \\
\hline 19 & 1.2242 & 133 & 19.967 & 17.603 & 20.437 & 141 \\
\hline 20 & 1.2255 & 145 & 19.655 & 17.517 & 19.093 & 132 \\
\hline 21 & 1.2256 & 143 & 19.681 & 17.509 & 17.947 & 124 \\
\hline 22 & 1.2258 & 125 & 17.293 & 17.495 & 17.837 & 128 \\
\hline 23 & 1.2260 & 142 & 20.807 & 17.475 & 19.863 & 146 \\
\hline 24 & 1.2264 & 138 & 20.496 & 17.447 & 15.450 & 111 \\
\hline 25 & 1.2269 & 125 & 16.902 & 17.413 & 17.207 & 118 \\
\hline 26 & 1.2277 & 140 & 19.384 & 17.358 & 21.297 & 145 \\
\hline 27 & 1.2281 & 108 & 14.658 & 17.333 & 19.296 & 129 \\
\hline 28 & 1.2294 & 136 & 19.935 & 17.242 & 14.921 & 105 \\
\hline 29 & 1.2295 & 124 & 17.069 & 17.236 & 19.872 & 135 \\
\hline 30 & 1.2300 & 128 & 17.973 & 17.207 & 18.011 & 123 \\
\hline 31 & 1.2302 & 128 & 18.594 & 17.189 & 17.003 & 116 \\
\hline 32 & 1.2302 & 119 & 16.009 & 17.187 & 17.521 & 129 \\
\hline 33 & 1.2303 & 128 & 18.984 & 17.180 & 18.083 & 132 \\
\hline 34 & 1.2307 & 125 & 17.453 & 17.157 & 17.693 & 125 \\
\hline 35 & 1.2313 & 127 & 17.617 & 17.117 & 17.708 & 129 \\
\hline 36 & 1.2322 & 121 & 17.901 & 17.059 & 17.297 & 122 \\
\hline 37 & 1.2326 & 143 & 19.657 & 17.032 & 16.448 & 123 \\
\hline 38 & 1.2326 & 128 & 17.987 & 17.031 & 18.130 & 125 \\
\hline 39 & 1.2336 & 122 & 17.154 & 16.963 & 17.194 & 127 \\
\hline 40 & 1.2343 & 116 & 15.852 & 16.918 & 16.316 & 112 \\
\hline
\end{tabular}




\section{References}

1. D. W. Boyd, 'Reciprocal polynomials having small measure'. Math. Comp. 35 (1980) 1361-1377. 126

2. D. W. Boyd, 'Speculations concerning the range of Mahler's measure', Canad. Math. Bull. 24 (1981) 453-469. 126

3. D. W. Boyd, 'Reciprocal polynomials having small measure II', Math. Comp. 53 (1989) 353-357, S1-S5. 126

4. D. W. Boyd, 'Mahler's measure and special values of $L$-functions', Experiment. Math. 7 (1998) 37-82. 126

5. C. Caldwell, 'Prime pages', http://www.utm.edu/research/primes. 128

6. V. СHотнi, G. Everest and T. WARD, ' $S$-integer dynamical systems: periodic points', J. Reine Angew. Math. 489 (1997) 99-132. 126

7. C. Deninger, 'Deligne periods of mixed motives, $K$-theory and the entropy of certain $\mathbb{Z}^{n}$-actions', J. Amer. Math. Soc. 10 (1997) 259-281. 126

8. G. EVEREST and T. WARD, Heights of polynomials and entropy in algebraic dynamics (Springer, London, 1999). 126, 126, 127

9. A. O. Gelfond, Transcendental and algebraic numbers (Dover, New York, 1960). 126

10. JoHn KNOPFMACHER, 'Arithmetical properties of finite rings and algebras, and analytic number theory. VI. Maximum orders of magnitude', J. Reine Angew. Math. 277 (1975) 45-62. 128

11. Edmund Landau, Handbuch der Lehre von der Verteilung der Primzahlen. 2 Bände, 2nd edn, with an appendix by Paul T. Bateman (Chelsea Publishing Co., New York, 1953). 128

12. D. H. LeHMER, 'Factorization of certain cyclotomic functions', Ann. of Math. 34 (1933) 461-479. 125

13. D. A. LIND, 'Dynamical properties of quasihyperbolic toral automorphisms', Ergodic Theory Dynam. Systems 2 (1982) 49-68. 126, 126

14. D. A. Lind, K. Schmidt and T. WARD, 'Mahler measure and entropy for commuting automorphisms of compact groups', Invent. Math. 101 (1990) 593-629. 126

15. K. MAHLER, 'An application of Jensen's formula to polynomials', Mathematika 7 (1960) 98-100. 126

16. M. J. Mossinghoff, 'Polynomials with small Mahler measure', Math. Comp. 67 (1998) 1697-1705. 126, 126, 126, 130, 131, 131

17. M. J. Mossinghoff, C. G. Pinner and J. D. VAaler, 'Perturbing polynomials with all their roots on the unit circle', Math. Comp. 67 (1998) 1707-1726. 126

18. 'PARI-GP', http://www.parigp-home.de. 130

19. T. A. Pierce, 'Numerical factors of the arithmetic forms $\prod_{i=1}^{n}\left(1 \pm \alpha_{i}^{m}\right)$ ', Ann. of Math. 18 (1917) 53-64. 125

20. Michael Rosen, 'A generalization of Mertens' theorem', J. Ramanujan Math. Soc. 14 (1999) 1-19. 128

21. C. J. Sмyтн, 'On the product of conjugates outside the unit circle of an algebraic integer', Bull. London Math. Soc. 3 (1971) 169-175. 126 
Primes in sequences associated to polynomials (after Lehmer)

22. S. S. WagstafF, 'Divisors of Mersenne numbers', Math. Comp. 40 (1983) 385-397. 128

Manfred Einsiedler manfred@mat.univie.ac.at

Mathematical Institute

University of Vienna

Strudlhofgasse 4

A-1090 Vienna

Austria

Graham Everest g.everest@uea.ac.uk

Thomas Ward t.wardeuea.ac.uk

School of Mathematics

University of East Anglia

Norwich NR4 7TJ 